\title{
Managing patients with acute type A aortic dissection and mesenteric malperfusion syndrome: A 20-year experience
}

\author{
Bo Yang, MD, PhD, ${ }^{\text {a }}$ Elizabeth L. Norton, MS, ${ }^{\mathrm{b}}$ Carlo Maria Rosati, MD, ${ }^{\mathrm{a}}$ Xiaoting Wu, PhD, ${ }^{\mathrm{a}}$ \\ Karen M. Kim, MD, ${ }^{a}$ Minhaj S. Khaja, MD, MBA, ${ }^{c}$ G. Michael Deeb, MD, ${ }^{a}$ David M. Williams, MD, ${ }^{c}$ and
} Himanshu J. Patel, MD ${ }^{\mathrm{a}}$

\begin{abstract}
Objective: To assess outcomes of endovascular reperfusion followed by delayed open aortic repair for stable patients with acute type A aortic dissection and mesenteric malperfusion syndrome (mesMPS).

Methods: Among 602 patients with acute type A aortic dissection who presented to our center from 1996 to 2017, all 82 (14\%) with mesMPS underwent upfront endovascular fenestration/stenting. Primary outcomes were in-hospital mortality and long-term survival. Patients with acute type A aortic dissection with no malperfusion syndrome of any organ $(n=419)$ served as controls.
\end{abstract}

Results: In-hospital mortality of all comers with mesMPS was 39\%. After endovascular fenestration/stenting, 20 mesMPS patients (24\%) died from organ failure and 11 patients $(13 \%)$ died from aortic rupture before open aortic repair, 47 patients $(58 \%)$ underwent aortic repair, and 4 patients (5\%) survived without open repair. No patients died from aortic rupture during the second decade (20082017). The significant risk factors for death from organ failure after endovascular reperfusion were acute stroke (odds ratio, 23; 95\% confidence interval, 4-144; $P=.0008$ ), gross bowel necrosis at laparotomy (odds ratio, $7 ; 95 \%$ confidence interval, 1.4-34; $P=.016$ ), and serum lactate $\geq 6 \mathrm{mmol} / \mathrm{L}$ (odds ratio, 13.5 ; $95 \%$ confidence interval, 2-97; $P=.0097)$. There was no significant difference in operative mortality $(2.1 \%$ vs $7.5 \% ; P=.50)$ or long-term survival between patients with mesMPS who underwent open aortic repair after recovering from mesMPS and patients with no malperfusion syndrome.

Conclusions: In patients with acute type A aortic dissection with mesMPS, endovascular fenestration/stenting, and delayed open aortic repair achieved favorable short- and long-term outcomes. Surgeons should consider correcting mesenteric malperfusion before undertaking open aortic repair in patients with mesMPS, especially those with acute stroke, gross bowel necrosis at laparotomy, or serum lactate $\geq 6$ mmol/L. (J Thorac Cardiovasc Surg 2019;158:675-87)

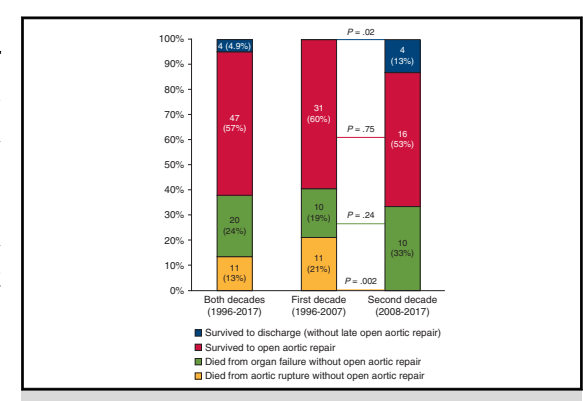

Short-term outcomes of patients with mesMPS after percutaneous endovascular reperfusion.

\section{Central Message}

Acute type A aortic dissection with mesenteric malperfusion syndrome should be treated with endovascular revascularization (ie, fenestration/stenting) and delayed open aortic repair to improve outcomes.

\section{Perspective}

Upfront endovascular fenestration/stenting in acute type A aortic dissection with mesenteric malperfusion syndrome can resolve visceral malperfusion quickly and adequately, and improve the chance of recovery from critical organ failure and survival with subsequent open aortic repair. This approach should be considered before open aortic repair in patients with mesenteric malperfusion syndrome.

See Commentaries on pages 688 and 690.
Acute type A aortic dissection (ATAAD) is a catastrophic event with an average operative mortality of $20 \%$ to $25 \%$. $^{1}$ Prompt surgical intervention (open aortic repair) is generally necessary to prevent death from aortic rupture.

\footnotetext{
From the Departments of ${ }^{\mathrm{a}}$ Cardiac Surgery and ${ }^{\mathrm{c}}$ Radiology, Michigan Medicine, Ann Arbor, Mich; and ${ }^{\mathrm{b}}$ Creighton University School of Medicine, Omaha, Neb.

Dr Yang is supported by the National Heart, Lung, and Blood Institute of the National Institutes of Health (grant No. R01HL141891, K08HL130614) and Phil Jenkins and Darlene \& Stephen J. Szatmari Funds. Dr Patel is supported by the Joe D. Morris Collegiate Professorship, the David Hamilton Fund, and the Phil Jenkins Breakthrough Fund in Cardiac Surgery. Dr Deeb is supported by the Herbert Sloan Collegiate Professorship, Jamie Buhr Fund, and Richard Nerod Fund.

Dr Yang and Ms Norton contributed equally as first authors.

Drs Patel, Williams, and Deeb contributed equally as senior authors.
}

Patients with ATAAD presenting with concomitant dissection-related severe end-organ malperfusion, tissue/ organ necrosis, and consequential failure (malperfusion syndrome [MPS]) have a significantly increased mortality.

\footnotetext{
Read at the 98th Annual Meeting of The American Association for Thoracic Surgery, San Diego, California, April 28-May 1, 2018.

Received for publication April 15, 2018; revisions received Oct 18, 2018; accepted for publication Nov 5, 2018; available ahead of print Jan 31, 2019.

Address for reprints: Bo Yang, MD, PhD, Department of Cardiac Surgery, Michigan Medicine, 1500 E Medical Center Dr, 5155 Frankel Cardiovascular Center, Ann Arbor, MI, 48109 (E-mail: boya@med.umich.edu) $0022-5223 / \$ 36.00$

Copyright $(2018$ by The American Association for Thoracic Surgery https://doi.org/10.1016/j.jtevs.2018.11.127
} 


\section{Abbreviations and Acronyms}

ATAAD = acute type A aortic dissection

$\mathrm{CPB}=$ cardiopulmonary bypass

IR = interventional radiology

mesMPS $=$ mesenteric malperfusion syndrome

MPS $=$ malperfusion syndrome

non-MPS $=$ no malperfusion syndrome

SMA $=$ superior mesenteric artery

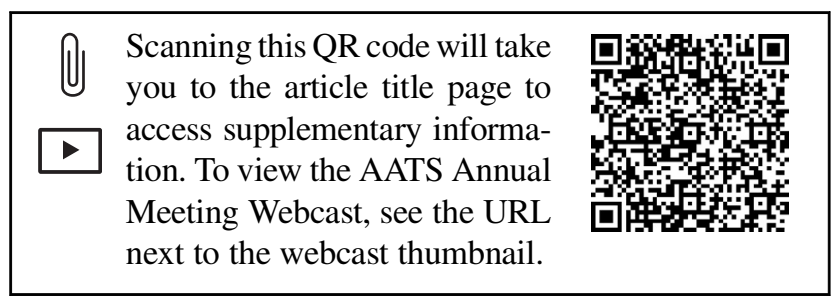

Mesenteric MPS (mesMPS), in particular, has been reported to have a very poor prognosis with in-hospital mortality ranging from $60 \%$ to $75 \% .^{1-12}$ The optimal management of patients with ATAAD with MPS remains an unsettled issue. Emergent open aortic repair aims to prevent aortic rupture and death from hemorrhagic shock or tamponade. However, for patients with mesMPS, the impending risk of bowel necrosis and septic shock might be the most immediate, life-threatening concern. Aortic repair usually achieves visceral reperfusion through surgical stabilization of the true lumen ${ }^{2}$ and resolves dynamic occlusion of the superior mesenteric artery (SMA), but it might fail in instances of static occlusion of the SMA, such as with SMA true or false lumen thrombosis. In addition, relief of the SMA obstruction does not cause immediate resolution of the already existing ischemic injury. In both cases, putting a patient through the major physiologic stress of a long operation on cardiopulmonary bypass with circulatory arrest before correction of or recovery from mesMPS could increase the probability of a poor outcome.

At the University of Michigan, since 1996 we have adopted a different approach for patients with ATAAD with MPS who are otherwise stable (ie, no aortic rupture and no tamponade), which consists of upfront endovascular reperfusion (through fenestration/stenting) of the critically malperfused organ system(s), including abdominal viscera, by interventional radiology (IR), followed by open aortic repair at resolution of organ failure. ${ }^{13-16}$ Herein, we present the 20-year experience of our approach, focusing on patients with ATAAD and mesMPS. The primary objective was to assess short- and long-term outcomes of ATAAD patients with mesMPS. A secondary objective was to identify risk factors for death from organ failure even after successful visceral reperfusion to differentiate patients for whom open aortic repair could achieve survival at discharge from those for whom it would likely be futile.

\section{METHODS}

This study was approved by the University of Michigan Institutional Review Board (Ann Arbor, Mich) and was in compliance with Health Insurance Portability and Accountability Act regulations. A waiver of consent was obtained.

\section{Patient Population and Data Collection}

Between July 1996 and January 2017, 602 patients presented to the University of Michigan with an ATAAD, defined as onset within 14 days of admission. Eighty-two patients (14\%) had mesMPS and represent the focus of this study. Patients ( $\mathrm{n}=419 ; 70 \%$ ), who did not have any (ie, cerebrospinal, coronary, mesenteric, renal, or lower extremity) MPS and underwent upfront open aortic repair, served as control patients (see Figure E1). Investigators obtained Society of Thoracic Surgery data elements from the University of Michigan Department of Cardiac Surgery Data Warehouse to determine pre-, intra-, and postoperative characteristics. Demographic characteristics, medical records, and operative reports were reviewed to supplement data collection. Survival was obtained through the National Death Index database through December $2015^{17}$ and medical record review.

\section{Diagnosis and Management of ATAAD With MesMPS}

The diagnosis of mesMPS requires both clinical and laboratory features (eg, abdominal pain, bloody diarrhea, tenderness to palpation, elevated lactate, and metabolic acidosis) as well as radiographic demonstration of low or absent blood flow through the SMA with or without SMA thrombosis. Unless there was evidence of aortic rupture or cardiac tamponade (indications for immediate aortic repair), all patients with mesMPS were treated with upfront endovascular fenestration/stenting before open repair. If the SMA was dissected, SMA pressure was measured beyond the distal extent of the dissection. Angiographic confirmation of treatable mesMPS was documented by a significant systolic blood pressure gradient $(>15 \mathrm{~mm} \mathrm{Hg}$ ) between the ascending aorta and the distal SMA. In patients with aortic coarctation, a blood pressure differential of $>20 \mathrm{~mm} \mathrm{Hg}$ has traditionally been considered notable, so we conservatively choose a gradient of systolic blood pressure of $15 \mathrm{~mm} \mathrm{Hg}$ as the criterion for malperfusion. Aortic fenestration and stenting were performed percutaneously by creating a tear in the dissection flap to balance the blood pressure and permit flow between the true and false lumens, as previously described. ${ }^{13-16}$ If the gradient between the ascending aorta and SMA persisted $(>15 \mathrm{~mm} \mathrm{Hg})$ after the aortic fenestration and stenting, further SMA stenting, thrombolysis, or suction thromboembolectomy was performed (Table E1). In dissected vessels with thrombosed false lumens, gradients after stenting might exceed $15 \mathrm{~mm} \mathrm{Hg}$, but as long as absolute perfusion pressure was viable; that is, $>60 \mathrm{~mm} \mathrm{Hg}$, postdilation of stents was not performed.

\section{Post-IR Management and Open Aortic Repair}

After endovascular reperfusion of the SMA, the general surgery team evaluated the 82 patients to determine whether an exploratory laparotomy was needed based on the clinical exam (abdominal tenderness), increasing lactate, and pneumatosis in the wall of the intestine on computed tomography scan (Figure 1). If no laparotomy was indicated or laparotomy was performed but no necrotic bowel was identified, we waited for resolution of abdominal pain, normalization of lactate and metabolic acidosis, and then performed open aortic repair. If a patient had a resection of necrotic bowel, we waited for the patient to recover from metabolic acidosis, septic shock, and/or acute respiratory distress 
syndrome, and then performed open aortic repair. The cohort of patients with mesMPS who eventually underwent open aortic repair after recovering from MPS was defined as surgical mesMPS patients $(n=47)$. The median time from fenestration/stenting to open aortic repair was 6 days (interquartile range, 2-19 days) (Table 1). The operative strategy for the surgical mesMPS patients was the same as for the patients with ATAAD with no MPS (non-MPS).

\section{Statistical Analysis}

The $\chi^{2}$ and Fisher exact tests were used for categorical variables and the Wilcoxon rank-sum test was used for continuous variables, as appropriate. Multivariable logistic regression was used to determine the risk factors with odds ratio (OR) for in-hospital mortality in comparison of all patients with mesMPS versus non-MPS after stepwise selection of variables, including group, age, gender, New York Heart Association functional class III or IV, history of myocardial infarction, previous cardiac surgery, coronary artery disease, chronic obstructive pulmonary disease, cardiogenic shock, acute myocardial infarction, acute stroke, acute renal failure, acute paralysis, and preoperative chronic renal failure based on clinical relevance. Multivariable logistic regression was also used to determine the risk factors and OR of mortality in comparison of patients who died from organ failure after endovascular reperfusion but before open repair versus those who survived to open repair or discharge. Stepwise selection was performed for variables, including age, acute stroke, renal malperfusion, extremity malperfusion, cardiogenic shock, bowel necrosis identified at laparotomy, and max serum lactate before IR $\geq 6 \mathrm{mmol} / \mathrm{L}$. Long-term survival curves were estimated using the Kaplan-Meier method with the log-rank test in all patients with mesMPS versus non-MPS patients, and surgical mesMPS patients versus non-MPS patients. The Cox proportional hazard model was used to compare the long-term outcome in surgical mesMPS patients versus non-MPS patients, but not in all patients with mesMPS versus non-MPS due to violation of proportional hazard assumption. Stepwise selection of variables was used for the Cox model, including all the variables used for the logistic model for in-hospital mortality. All statistical analysis was performed using SAS version 9.4 (SAS Institute Inc, Cary, NC).

\section{RESULTS \\ Demographic Characteristics of Patients With MesMPS Versus Non-MPS}

As expected, patients with mesMPS were much sicker than non-MPS patients when they arrived at our hospital, including a significantly higher incidence of acute stroke, acute renal malperfusion, acute kidney injury with or without dialysis, acute extremity malperfusion, acute paralysis, bowel necrosis, chronic renal failure, hypertension, and previous myocardial infarction and cardiac surgery (Table 1).

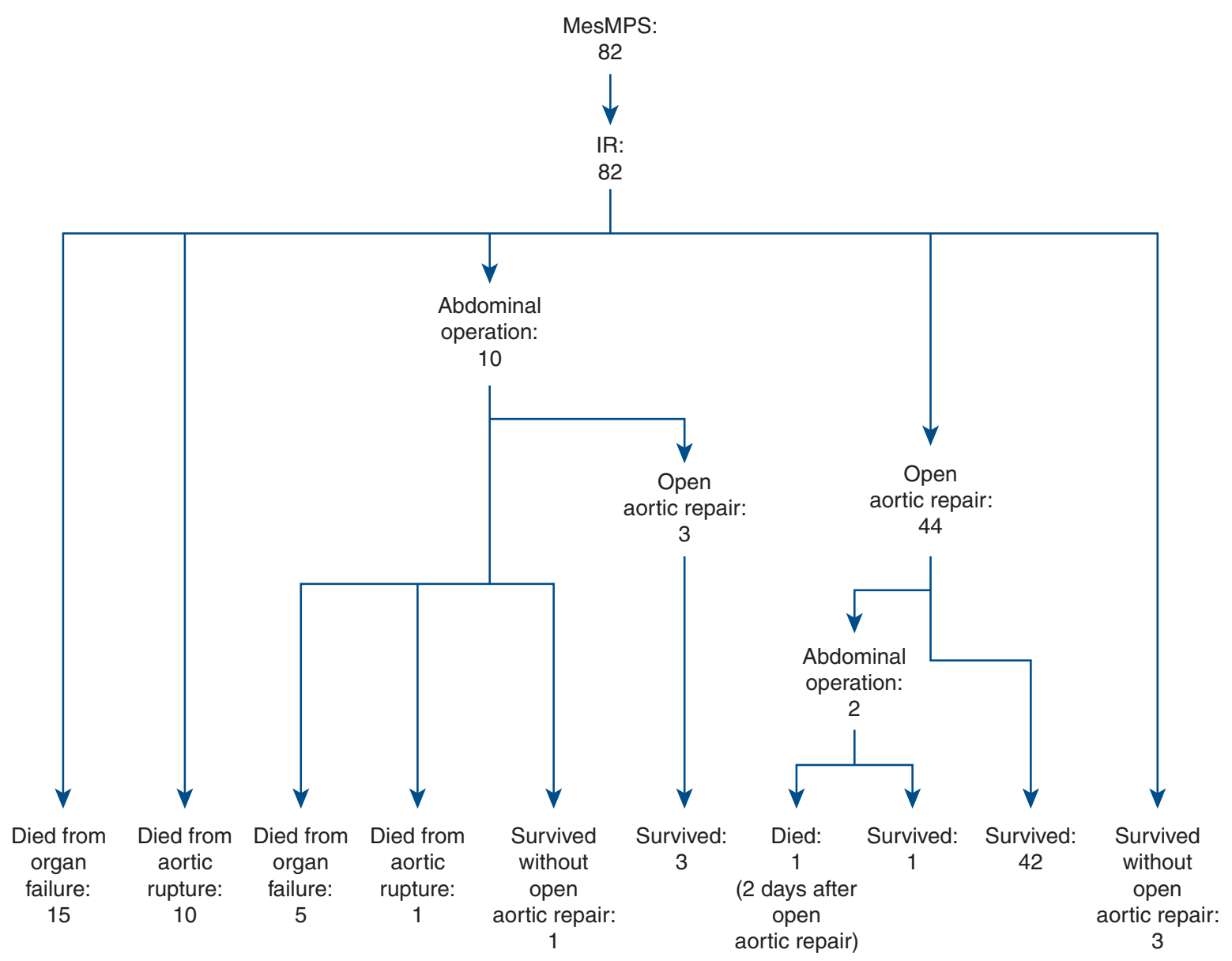

FIGURE 1. Management and short-term outcomes of patients with mesenteric malperfusion syndrome. MesMPS, Mesenteric malperfusion syndrome; IR, endovascular reperfusion by interventional radiology. 
TABLE 1. Demographic and preoperative characteristics of all patients

\begin{tabular}{|c|c|c|c|c|}
\hline Admission variable & $\frac{\text { All patients }}{(\mathrm{N}=501)}$ & $\begin{array}{c}\text { MesMPS } \\
(\mathbf{n}=\mathbf{8 2})\end{array}$ & $\begin{array}{l}\text { Non-MPS } \\
(n=419)\end{array}$ & $P$ value \\
\hline Age on admission (y) & $59(49-68)$ & $59.5(50-68)$ & $59(49-68)$ & .97 \\
\hline Female gender & $151(30)$ & $22(27)$ & $129(31)$ & .48 \\
\hline NYHA functional class III or IV & $99(22)$ & $8(10)$ & $91(22)$ & .013 \\
\hline CAD & $88(18)$ & $17(20)$ & $71(17)$ & .41 \\
\hline History of MI & $29(5.8)$ & $9(11)$ & $20(4.8)$ & .03 \\
\hline Previous cardiac surgery & $50(10)$ & $13(16)$ & $37(8.8)$ & .04 \\
\hline Hypertension & $351(70)$ & $65(81)$ & $286(68)$ & .019 \\
\hline COPD & $44(8.8)$ & $7(8.5)$ & $37(8.8)$ & .93 \\
\hline Smoking status & & & & .96 \\
\hline Never smoker & $232(47)$ & $36(46)$ & $196(47)$ & \\
\hline Former smoker & $126(25)$ & $20(25)$ & $106(25)$ & \\
\hline Current smoker & $138(28)$ & $23(29)$ & $115(28)$ & \\
\hline Diabetes & $27(5.5)$ & $2(2.7)$ & $25(5.9)$ & .40 \\
\hline Creatinine on admission (mg/dL) & $1.0(0.8-1.3)$ & $1.4(1.0-2.3)$ & $1.0(0.8-1.2)$ & $<.0001$ \\
\hline Chronic kidney disease & $19(3.8)$ & $8(9.9)$ & $11(2.6)$ & .006 \\
\hline History of CVA & $14(2.8)$ & $3(3.7)$ & $11(2.6)$ & .71 \\
\hline PVOD & $57(11)$ & $9(11)$ & $48(11)$ & .90 \\
\hline Connective tissue disorder & $28(5.6)$ & $2(2.4)$ & $26(6.2)$ & .29 \\
\hline Aortic insufficiency & & & & .97 \\
\hline None & $130(28)$ & $21(28)$ & $109(28)$ & \\
\hline Trace/trivial & $54(12)$ & $10(13)$ & $44(11)$ & \\
\hline Mild & $94(20)$ & $15(20)$ & $79(20)$ & \\
\hline Moderate & $83(18)$ & $14(19)$ & $69(18)$ & \\
\hline Severe & $104(22)$ & $15(20)$ & $89(23)$ & \\
\hline Cardiogenic shock & $38(7.8)$ & $3(4.3)$ & $35(8.4)$ & .23 \\
\hline Acute stroke & $12(2.4)$ & $10(12)$ & $2(0.5)$ & $<.0001$ \\
\hline Acute paralysis & $6(1.2)$ & $6(7.3)$ & $0(0)$ & $<.0001$ \\
\hline Acute MI & $1(0.2)$ & $1(1.2)$ & $0(0)$ & .16 \\
\hline Tamponade & $42(8.4)$ & $3(3.7)^{*}$ & $39(9.3)$ & .09 \\
\hline AKI & $80(16)$ & $62(76)$ & $18(4.3)$ & $<.0001$ \\
\hline Requiring new dialysis preoperatively & $5(1.0)$ & $5(6.1)$ & $0(0)$ & .0001 \\
\hline Renal malperfusion & $55(11)$ & $55(67)$ & $0(0)$ & \\
\hline Lower extremity malperfusion & $53(11)$ & $53(65)$ & $0(0)$ & \\
\hline Bowel necrosis at laparotomy & $9(1.8)$ & $9(11)$ & $0(0)$ & \\
\hline Max serum lactate before IR (mmol/L) & NA & $2.3(1.2-3.4)$ & NA & \\
\hline Max serum lactate before $\mathrm{IR} \geq 6 \mathrm{mmol} / \mathrm{L}$ & NA & $7(8.5)$ & NA & \\
\hline \multicolumn{5}{|l|}{ Management } \\
\hline IR & $82(16)$ & $82(100)$ & $0(0)$ & $<.001$ \\
\hline Time from admission to IR (d) & NA & $0(0-1)$ & NA & NA \\
\hline Open aortic repair & $466(93)$ & $47(57)$ & $419(100)$ & $<.001$ \\
\hline Time from admission to aortic repair (d) & $0(0-1)$ & $7(3-20)$ & $0(0-1)$ & $<.0001$ \\
\hline Time from IR to aortic repair (d) & NA & $6(2-19)$ & NA & NA \\
\hline
\end{tabular}

Values are presented as median (interquartile range) for continuous variables and number (\%) for categorical variables. mesMPS, Malperfusion syndrome; non-MPS, no malperfusion syndrome; $N Y H A$, New York Heart Association; $C A D$, coronary artery disease; $M I$, myocardial infarction; $C O P D$, chronic obstructive pulmonary disease; $C V A$, cerebrovascular accident; $P V O D$, peripheral vascular occlusive disease; $A K I$, acute kidney injury; $I R$, endovascular procedure by interventional radiology; $N A$, not applicable.

*Three patients developed cardiac tamponade after IR procedure due to progression of disease. 


\section{Overall Outcomes of Patients With ATAAD and MesMPS}

All 82 patients with ATAAD and mesMPS underwent upfront endovascular reperfusion by IR (Figure 1 and Table E1). After endovascular fenestration/stenting, 38\% of patients died before open aortic repair either from organ failure $(\mathrm{n}=20 ; 24.4 \%)$ or aortic rupture $(\mathrm{n}=11 ; 13.4 \%)$. The remaining patients survived and either underwent an open aortic repair ( $\mathrm{n}=47$ [57\% ]; 44 patients underwent an operation before discharge and 3 patients had an operation at readmission within 1 year) or survived through discharge but never had an open aortic repair $(n=4 ; 5 \%)$ (Figure 2$)$. All deaths from aortic rupture $(\mathrm{n}=11)$ occurred during the first decade (1996-2007), with 0 deaths from aortic rupture in the second decade (2008-2017) $(P=.002)$ (Figure 2). One patient died after open aortic repair. The overall in-hospital mortality was $39 \%$ (Table 2 ).

\section{Risk Factors for Death From Organ Failure After Resolution of Malperfusion}

The patients with mesMPS who died from organ failure after endovascular revascularization had significantly more cardiogenic shock, acute stroke, and higher lactate levels compared with those who survived to open aortic repair or discharge (Table 3). Multivariable logistic regression showed the significant independent risk factors for

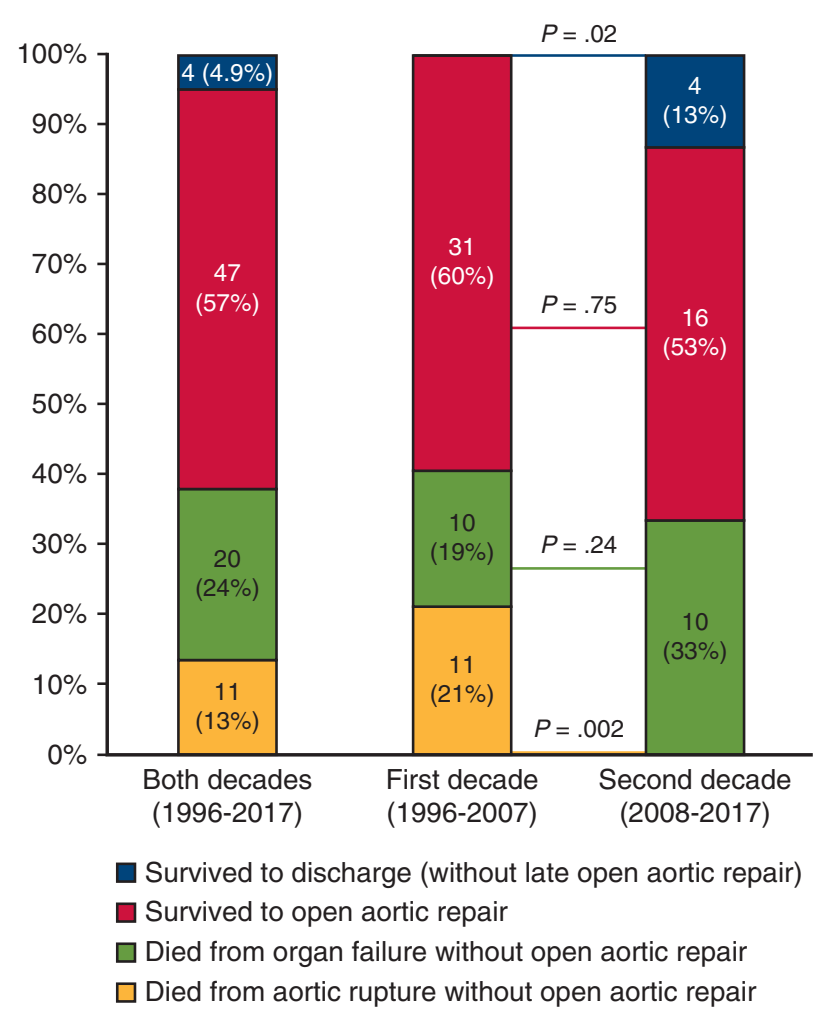

FIGURE 2. Short-term outcomes of patients with mesenteric malperfusion syndrome after endovascular reperfusion by interventional radiology. death from organ failure were acute stroke (OR, 23; 95\% confidence interval $[\mathrm{CI}], 4-144 ; P=.0008$ ), gross bowel necrosis at laparotomy (OR, 7; 95\% CI, 1.4-34); $P=.016$ ), and serum lactate $\geq 6 \mathrm{mmol} / \mathrm{L}$ (OR, 13.5; 95\% CI, 2-97; $P=.0097)$.

\section{Short-Term Outcomes of Patients With MesMPS Versus Non-MPS}

The in-hospital mortality was 5-times higher in the mesMPS group $(\mathrm{n}=82)$ compared with the non-MPS group $(\mathrm{n}=419)(39 \%$ vs $7.2 \% ; P<.001)$. All other postprocedure outcomes (IR or open repair) in the mesMPS group were not significantly different from the postoperative outcomes in the non-MPS group despite the fact that patients with mesMPS were much sicker at admission (Table 2). Multivariable logistic regression showed the significant risk factors for in-hospital mortality were mesMPS (OR, 5; 95\% CI, 2.4-10.6; $P<.0001)$, age (OR, 1.03; 95\% CI, $1-1.05 ; P=.046)$, cardiogenic shock (OR, 8; 95\% CI, $3.5-14 ; P<.001)$, and acute stroke (OR, 12; 95\% CI, 2.6$59 ; P=.002)$.

\section{Comparison of Surgical Outcomes Between Surgical MesMPS and Non-MPS Patients}

Although surgical mesMPS patients were still significantly sicker at admission (Table E2), there was no significant difference in the complexity of the operation (Table E3) or the operative mortality (30-day postoperative or inhospital) $(2.1 \%$ vs $7.4 \% ; P=.23)$ between the surgical mesMPS versus non-MPS groups (Table 4). However, surgical mesMPS patients required more blood transfusions intraoperatively (Table E3), and had longer postoperative (after open aortic repair) hospital stays (Table 4).

\section{Long-Term Survival}

Patients with mesMPS $(n=82)$ had worse overall longterm survival than non-MPS patients due to a much higher in-hospital mortality (10-year survival, $41 \%$; $95 \%$ CI, $29 \%-53 \%$ vs $64 \%, 95 \%$ CI, $57 \%-69 \% ; P<.001)$ (Figure 3, A) However, there was no significant difference in long-term survival between surgical patients with mesMPS ( $\mathrm{n}=47)$ and non-MPS patients (10-year survival, $69 \%$; $95 \%$ CI, $50 \%-82 \%$ ) vs $64 \%$; $95 \%$ CI, $57 \%-69 \%$; $P=.60$ ) (Figure $3, B$ ) Cox proportional hazard model showed that the risk factors for all-time mortality after surgery in surgical mesMPS patients and non-MPS patients were age (hazard ratio [HR], 1.04; 95\% CI, 1.02-1.05; $P<.001)$, CAD (HR, $1.8 ; 95 \% \mathrm{CI}, 1.3-2.7 ; P=.001)$, and acute paralysis (HR, 7.3; 95\% CI, 1.6-33; $P=.01$ ). MesMPS at admission was no longer a risk factor after patients were successfully treated with endovascular fenestration/stenting and recovered from MPS (HR, $0.8 ; 95 \% \mathrm{CI}$, $0.4-1.4 ; P=.37)$. 
TABLE 2. Outcomes after procedure by interventional radiology (IR) or open repair (OR) of patients with mesenteric malperfusion syndrome (mesMPS) or no malperfusion syndrome (non-MPS)

\begin{tabular}{|c|c|c|c|c|}
\hline & $\begin{array}{l}\text { All patients } \\
(\mathbf{N}=\mathbf{5 0 1})\end{array}$ & $\begin{array}{c}\text { MesMPS } \\
(\mathbf{n}=\mathbf{8 2})\end{array}$ & $\begin{array}{l}\text { Non-MPS } \\
(\mathrm{n}=419)\end{array}$ & $P$ value \\
\hline Reoperation for bleeding & $43(8.6)$ & $5(6.1)$ & $38(9.1)$ & .37 \\
\hline Tamponade & $10(2.0)$ & $0(0)$ & $10(2.4)$ & .38 \\
\hline Postoperative MI & $8(1.6)$ & $2(2.4)$ & $6(1.4)$ & .62 \\
\hline Atrial fibrillation & $165(33)$ & $24(29)$ & $141(34)$ & .43 \\
\hline New-onset CVA & $33(6.6)$ & $3(3.7)$ & $30(7.2)$ & .24 \\
\hline New-onset paraplegia & $3(0.6)$ & $1(1.2)$ & $2(0.5)$ & .42 \\
\hline Pneumonia & $82(16)$ & $17(21)$ & $65(16)$ & .25 \\
\hline Reintubation & $28(5.6)$ & $4(4.9)$ & $24(5.7)$ & 1.0 \\
\hline Tracheostomy & $17(3.4)$ & $14(3.4)$ & $3(3.7)$ & .75 \\
\hline Postoperative AKI & $46(9.2)$ & $3(3.7)$ & $43(10)$ & .06 \\
\hline Requiring new dialysis & $19(3.8)$ & $1(1.2)$ & $18(4.3)$ & .34 \\
\hline Total LOS (d) & $11(7-18)$ & $18(4-28)$ & $10(7-16)$ & .12 \\
\hline In-hospital mortality & $62(12)$ & $32(39)$ & $30(7.2)$ & $<.0001$ \\
\hline
\end{tabular}

Values are presented as median (interquartile range) for continuous variables and $\mathrm{n}(\%)$ for categorical variables. In the mesMPS group, any complications post-IR procedures or post-OR were recorded as outcomes. In the non-MPS group, any complications post-OR were recorded as outcomes. mesMPS, Malperfusion syndrome; non-MPS, no malperfusion syndrome; $M I$, Myocardial infarction; $C V A$, cerebrovascular accident; $A K I$, acute kidney injury; $L O S$, length of stay.

\section{DISCUSSION}

In this study, we reported our 20-year experience of managing ATAAD with MesMPS treated with endovascular reperfusion and delayed open aortic repair. The key findings include in-hospital mortality for all patients with mesMPS (after endovascular reperfusion, with or without open repair) was $39 \%$, which was 5 times higher than that in non-MPS patients; $24 \%$ of patients with mesMPS died as a result of organ failure despite adequate endovascular revascularization (the notable risk factors of death from organ failure were acute stroke, gross bowel necrosis at laparotomy, and maximum lactate $\geq 6 \mathrm{mmol} / \mathrm{L}$ ); and after endovascular revascularization and recovery from mesMPS, the postoperative short- and long-term survival in surgical mesMPS patients was not significantly different from that in non-MPS patients (Video 1).

There are 2 critical issues in patients with ATAAD and mesMPS: rupture of the proximal aorta and mesenteric ischemia/necrosis, which can both result in death. Traditional teaching predicates immediate open aortic repair for all patients with ATAAD, even with mesMPS, with the goal to protect the patient from aortic rupture and to resolve the mesenteric malperfusion. This strategy may work for dynamic obstruction of the SMA if no end-organ damage (ie, MPS) has occurred; however, once end-organ damage is present or static occlusion of the SMA due to thrombosis of the false lumen in the SMA is present, the mesenteric issue cannot reliably be resolved by open aortic repair alone. More than $50 \%$ of patients with mesMPS in our study needed SMA stenting, focal thrombolysis, or suction thromboembolectomy to resolve the obstruction of the SMA (Table E1). Some institutions perform SMA stenting after open repair if patients were suspected to have ongoing mesenteric ischemia and malperfusion. ${ }^{18}$ However, this approach could be too late if patients already have compromised bowel upon arrival. With the traditional approach, the operative mortality in patients with ATAAD with mesMPS is $>50 \%{ }^{1-12}$

Due to this limitation of the traditional approach (upfront open aortic repair), we developed our strategy to treat mesMPS with initial endovascular fenestration/stenting followed by open aortic repair upon recovery from mesMPS. There are several advantages to such an approach. First, we are able to resolve the obstruction of the SMA and other visceral branches immediately and adequately with a minimally invasive percutaneous procedure, avoiding cardiopulmonary bypass and hypothermic circulatory arrest. The procedure itself has minimal negative influence due to trauma on these very sick patients. In addition, we can treat SMA occlusion with stenting, thrombolysis with local fibrinolysis (tissue plasminogen activator), or suction thromboembolectomy (Table E1). At the end of the procedure, we measure the blood pressure in each visceral branch (such as the SMA, celiac artery, and renal arteries) and in the iliac arteries to confirm that arterial obstruction has been adequately relieved. We accept a blood pressure gradient between the ascending aorta and arterial branch below $15 \mathrm{~mm} \mathrm{Hg}$. Second, our approach avoids futile 
TABLE 3. Clinical condition of patients with mesenteric malperfusion syndrome (mesMPR) divided into 3 groups based on the outcome of endovascular reperfusion

\begin{tabular}{|c|c|c|c|c|}
\hline Variable & $\begin{array}{l}\text { Death from organ } \\
\text { failure }(n=20)\end{array}$ & Survival* $^{*}(\mathbf{n}=\mathbf{5 1})$ & $\begin{array}{l}\text { Death from aortic } \\
\text { rupture }(n=11)\end{array}$ & $P$ value $\dagger$ \\
\hline Age on admission (y) & $58(47-73)$ & $57(51-65)$ & $63(58-69)$ & .65 \\
\hline Female gender & $8(40)$ & $12(24)$ & $2(18)$ & .27 \\
\hline CAD & $5(28)$ & $11(22)$ & $1(10)$ & .11 \\
\hline History of MI & $4(21)$ & $4(7.8)$ & $1(10)$ & .20 \\
\hline Previous cardiac surgery & $3(15)$ & $9(18)$ & $1(9.1)$ & 1 \\
\hline Hypertension & $16(84)$ & $44(86)$ & $5(50)$ & 1 \\
\hline COPD & $1(5.0)$ & $5(9.8)$ & $1(9.1)$ & .67 \\
\hline Diabetes & $0(0)$ & $2(3.9)$ & $0(0)$ & 1 \\
\hline Creatinine on admission (mg/dL) & $1.4(1.1-2.5)$ & $1.4(1.0-2.6)$ & $1.3(1.1-1.4)$ & .88 \\
\hline GFR on admission $\left(\mathrm{mL} / \mathrm{min} / 1.73 \mathrm{~m}^{2}\right)$ & $67(42-93)$ & $69(47-96)$ & $62(48-92)$ & .98 \\
\hline Chronic kidney disease & $3(16)$ & $5(9.8)$ & $0(0)$ & .67 \\
\hline History of CVA & $0(0)$ & $3(5.9)$ & $0(0)$ & .55 \\
\hline PVOD & $2(10)$ & $7(14)$ & $0(0)$ & 1 \\
\hline Cardiogenic shock & $3(20)$ & $0(0)$ & $0(0)$ & .01 \\
\hline Acute stroke & $8(40)$ & $2(3.9)$ & $0(0)$ & $<.001$ \\
\hline Acute paralysis & $2(10)$ & $4(7.8)$ & $0(0)$ & 1 \\
\hline Acute MI & $0(0)$ & $0(0)$ & $1(9.1)$ & 1 \\
\hline Preoperative CPR & $0(0)$ & $1(2.0)$ & $0(0)$ & 1 \\
\hline $\begin{array}{l}\text { AKI } \\
\text { Requiring new dialysis preoperatively }\end{array}$ & $\begin{array}{r}16(80) \\
1(5.0)\end{array}$ & $\begin{array}{r}38(75) \\
4(7.8)\end{array}$ & $\begin{array}{l}8(73) \\
0(0)\end{array}$ & $\begin{array}{l}.76 \\
1.0\end{array}$ \\
\hline Renal malperfusion & $15(75)$ & $33(65)$ & $7(64)$ & .65 \\
\hline Lower extremity malperfusion & $11(55)$ & $36(71)$ & $6(55)$ & .33 \\
\hline Bowel necrosis at laparotomy & $5(25)$ & $4(7.8)$ & $0(0)$ & .12 \\
\hline Max serum lactate before IR (mmol/L) & $3.0(2.2-6.8)$ & $1.6(1.2-3.2)$ & $2.2(1.6-2.7)$ & .02 \\
\hline Max serum lactate before $\mathrm{IR} \geq 6 \mathrm{mmol} / \mathrm{L}$ & $5(31)$ & $2(5)$ & $0(0)$ & .02 \\
\hline
\end{tabular}

Values are presented as n (\%) or median (range). CAD, Coronary artery disease; $M I$, myocardial infarction; COPD, chronic obstructive pulmonary disease; $G F R$, glomerular filtration rate as estimated using the Cockcroft-Gault formula; $C V A$, cerebrovascular accident; $P V O D$, peripheral vascular occlusive disease; $C P R$, cardiopulmonary resuscitation; $A K I$, acute kidney injury; $I R$, endovascular procedure by interventional radiology. *Patients survived to open aortic repair or discharge without open repair. †The difference between the groups of death from organ failure and survival to open aortic repair or hospital discharge.

open aortic repair, which may consume more resources than a percutaneous procedure alone. Twenty patients in this cohort died from organ failure even after arterial obstruction of all the viscera and extremities was resolved (Figures 1 and 2, and Table E4). Assuming an open aortic repair could have resolved the malperfusion, it is very likely that these patients would have died from organ failure due to the prolonged period of mesMPS. Third, we were able to treat all the patients with mesMPS with this approach even if they had mesenteric malperfusion for longer than 24 hours, as long as they did not have aortic rupture or cardiac tamponade. We do not turn down any patients with mesMPS no matter how sick they are. We give every patient a chance of recovery by reperfusion of his or her mesentery. Finally, our approach provides very favorable long-term survival in patients with mesMPS after endovascular reperfusion and subsequent open aortic repair, which was very similar to patients with ATAAD without MPS (Figure 3, $B$ ).

There is a difference between mesenteric malperfusion and mesMPS. Malperfusion is inadequate blood flow to an end organ; that is, ongoing arterial obstruction. The organ could be ischemic but not necrotic, especially at the early stage. MPS is the late stage of malperfusion and the malperfused end organ already has cell/tissue/organ death and malfunction, such as necrotic gut for mesenteric malperfusion. MPS is the indication for IR evaluation and possible treatment, but radiographic (such as CT angiogram) malperfusion alone is not. The patients who died 
TABLE 4. Postoperative outcomes of patients with mesenteric malperfusion syndrome (mesMPS) or without malperfusion syndrome (non-MPS) (only patients who underwent open aortic repair)

\begin{tabular}{|c|c|c|c|c|}
\hline Outcome & $\begin{array}{l}\text { All patients } \\
(\mathrm{N}=\mathbf{4 6 6})\end{array}$ & $\begin{array}{c}\text { MesMPS } \\
(\mathrm{n}=47) \\
\end{array}$ & $\begin{array}{c}\text { Non-MPS } \\
(n=419) \\
\end{array}$ & $P$ value \\
\hline Reoperation for bleeding & $43(9.2)$ & $5(11)$ & $38(9.1)$ & .79 \\
\hline Tamponade & $10(2.1)$ & $0(0)$ & $10(2.3)$ & .61 \\
\hline Perioperative MI & $6(1.2)$ & $0(0)$ & $6(1.4)$ & 1.0 \\
\hline Atrial fibrillation & $162(35)$ & $21(45)$ & $141(34)$ & .14 \\
\hline DSWI & $12(2.5)$ & $0(0)$ & $12(2.8)$ & .62 \\
\hline New-onset CVA & $33(7.1)$ & $3(6.4)$ & $30(7.2)$ & 1.0 \\
\hline New-onset paraplegia & $2(0.4)$ & $0(0)$ & $2(0.5)$ & 1.0 \\
\hline Pneumonia & $75(16)$ & $10(21)$ & $65(16)$ & .31 \\
\hline Reintubation & $27(5.8)$ & $3(6.4)$ & $24(5.7)$ & .75 \\
\hline Tracheostomy & $14(3.0)$ & $0(0)$ & $14(3.3)$ & .38 \\
\hline Postoperative AKI & $45(9.7)$ & $2(4)$ & $43(10)$ & .29 \\
\hline Requiring new dialysis & $19(4)$ & $1(2)$ & $18(4)$ & .71 \\
\hline Postoperative LOS (d) & $10(7-16)$ & $16(10-23)$ & $10(7-15)$ & $<.0001$ \\
\hline Intraoperative mortality & $4(0.8)$ & $0(0)$ & $4(0.9)$ & 1.0 \\
\hline In-hospital mortality & $31(6.7)$ & $1(2.1)$ & $30(7.2)$ & .35 \\
\hline 30-d mortality & $25(5.4)$ & $1(2.1)$ & $24(5.7)$ & .49 \\
\hline Operative mortality* & $32(6.9)$ & $1(2.1)$ & $31(7.4)$ & .23 \\
\hline
\end{tabular}

Values are presented as median (interquartile range) for continuous variables and $\mathrm{n}(\%)$ for categorical variables. mesMPS, Malperfusion syndrome; non-MPS, no malperfusion syndrome; $M I$, Myocardial infarction; $D S W I$, deep sternal wound infection; $C V A$, cerebrovascular accident; $A K I$, acute kidney injury; $L O S$, length of stay. *Defined as in-hospital mortality or mortality within 30 days after open repair.

from organ failure after endovascular revascularization in our study all had very-late-stage malperfusion and unsalvageable end-organ death, even after the mesenteric malperfusion was resolved by IR. Many of them had multiorgan arterial obstruction (Table E4). Any intervention most likely would have been futile, including IR and open repair. MesMPS includes all those patients with existing bowel damage and reperfusion injury, which are not eliminated by restoring arterial perfusion. However, when patients with MPS (late stage of malperfusion) come to us, we never know who will survive and who will not survive after revascularization. IR is less traumatic than open aortic repair, and at least gives those patients a chance to recover; maybe a better chance in those borderline patients due to much less trauma on the patients. This is why we recommend IR first for patients with MPS, especially because the risk of aortic rupture decreased dramatically with our current management.

A similar approach to treatment of patients with ATAAD with mesMPS has also been adapted by other groups, either through an endovascular approach ${ }^{10,19}$ or open SMA bypass $^{20,21}$ with good outcomes. Driven by the concern for impending aortic rupture, those groups performed open aortic repair immediately after mesenteric reperfusion. The reasons to delay open aortic repair in our approach are as follows: patients may have a necrotic bowel requiring bowel resection, patients may need time to recover from multiorgan failure, such as acute respiratory distress syndrome or septic shock from necrotic bowel, and some patients may die from organ failure even after visceral and/or extremity malperfusion are resolved (as we saw in our study). For these patients, immediate open aortic repair would be futile.

There is always the risk of proximal aortic rupture and death from hemorrhage or tamponade while waiting for delayed open aortic repair during recovery from mesenteric ischemia. Indeed, we had 11 patients $(13 \%)$ die from aortic rupture. All the cases of aortic rupture occurred in the first decade (1996-2007). As we gained more experience managing patients with ATAAD after endovascular fenestration/ stenting, we had no deaths from aortic rupture during the second decade (2008-2017) (Figure 2), likely due to aggressive blood pressure control (goal was systolic $<110 \mathrm{~mm}$ $\mathrm{Hg}$ ), better care in the intensive care unit, and earlier secondary open aortic repair (median waiting time, 7 days in the first decade vs 4 days in the second decade; $P=.44$ ).

To better predict the outcomes of patients with ATAAD with mesMPS, we aimed to identify variables associated with mortality from organ failure even after successful endovascular visceral reperfusion. We found that acute stroke, 


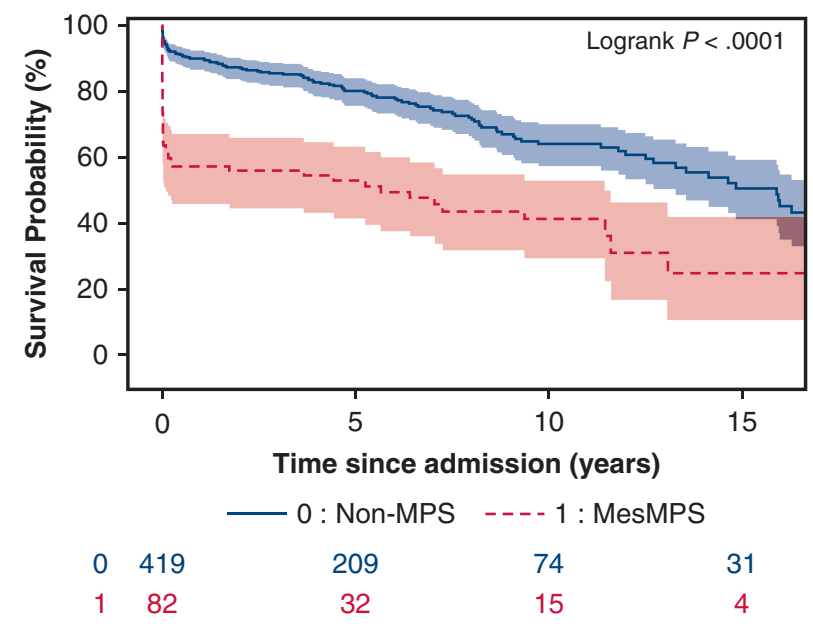

A



B

FIGURE 3. Overall long-term survival of patients with mesenteric malperfusion syndrome (MesMPS) versus those with no (mesenteric or nonmesenteric) malperfusion syndrome (Non-MPS). A, Survival since hospital admission, all MesMPS patients $(\mathrm{n}=82)$ versus non-MPS patients. B, Survival since open aortic repair, surgical MPS patients $(n=47)$ who underwent open aortic repair versus non-MPS patients.

gross bowel necrosis at laparotomy, and a serum lactate $\geq 6 \mathrm{mmol} / \mathrm{L}$ were significant independent risk factors of death from organ failure with a high odds ratio (7-23), which are consistent with other reports. ${ }^{22-24}$ Therefore, it might be valuable for the treating clinician to gauge the appropriateness versus futility of open aortic repair when patients present with an acute stroke, bowel necrosis, or high serum lactate in the setting of ATAAD with mesMPS.

The patients with mesMPS who recovered from endovascular reperfusion and subsequently underwent open aortic repair are a highly selected group. This group of patients had no more visceral or extremity malperfusion and had recovered from most complications (except renal failure) of mesMPS by the time they underwent open aortic repair.

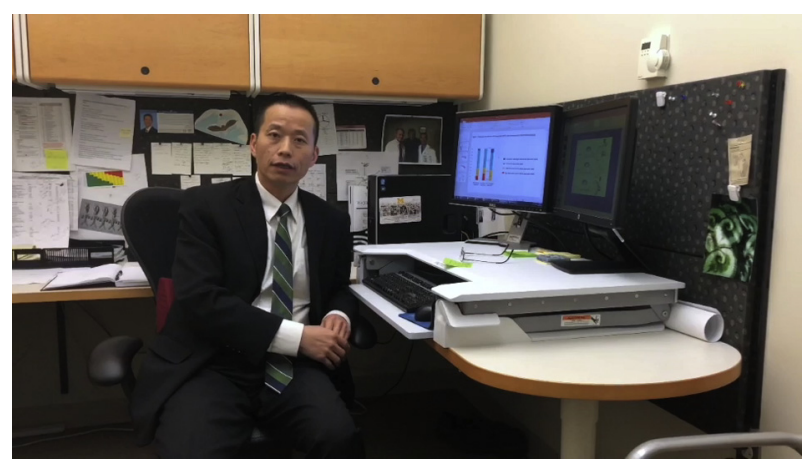

VIDEO 1. Discussion of the management of patients with acute type A aortic dissection with mesenteric malperfusion syndrome, including indications for upfront endovascular revascularization, detailed technique of fenestration and stenting of the dissected aorta and its branches, timing for delayed open aortic repair, and short- and long-term outcomes. Video available at: https://www.jtcvs.org/article/S0022-5223(18)33284-7/fulltext.

It is not surprising that the short- and long-term outcomes were comparable to non-MPS ATAAD patients (Table 4 and Figure 3,B). Actually, this is exactly our goal of treating patients with ATAAD with mesMPS: Reperfuse abdominal viscera as soon as possible, essentially converting a patient with mesMPS to a non-MPS patient, and improve survival in this difficult-to-treat disease.

This study is limited by a single-center retrospective experience, although, we report among the largest cohorts of patients with ATAAD with mesMPS. Because the severity of mesMPS would be expected to correlate with duration of SMA obstruction, it might have been helpful to include duration of symptoms as a variable associated with mortality from organ failure. Although serum lactate might be a surrogate for duration of SMA obstruction, lactate might be falsely low in the setting where superior mesenteric venous outflow is reduced due to celiac artery and SMA obstruction. Our institution is among only a few centers using this approach for patients with ATAAD with mesMPS, wherein the mesenteric arterial occlusion is corrected and the patient is allowed to recover from malperfusion before open aortic repair. Our results may not be reproducible at other institutions. The sample size of patients who died from organ failure before open repair is still small, which prevented us, for instance, from building a robust multivariable quantitative prognostic score for risk of death from organ failure.

\section{CONCLUSIONS}

In patients with ATAAD with mesMPS, endovascular fenestration/stenting followed by delayed open repair achieved favorable short- and long-term outcomes. For patients with mesMPS with acute stroke, bowel necrosis at laparotomy, or serum lactate $\geq 6 \mathrm{mmol} / \mathrm{L}$, the risk of dying from organ failure increased dramatically, and therefore caution should be exercised when offering open aortic 
repair to those patients. Surgeons should keep in mind that not every untreated ATAAD will rupture, but every untreated mesMPS will cause a patient's death.

\section{Webcast}

You can watch a Webcast of this AATS meeting presentation by going to: https://aats.blob.core.windows.net/ media/18Apr30/20ABC $\% 202$.Adult $\% 20$ Cardiac $\% 20$ SS/ S62_4.mp4.



\section{Statement of Potential Conflict of Interest}

Dr Williams is on the Medical Advisory Board of Boston Scientific and Drs Williams and Patel are consultants with Gore on an unrelated device. All other authors have nothing to disclose with regard to commercial support.

The authors thank the Data Warehouse, Department of Cardiac Surgery (led by Dr Donald Likosky and including Jeremy Wolverton, Amy Geltz, Mary Barry, Mary Ryzak, Brett Cross, and other team members) as well as the Department of Cardiac Surgery and the Frankel Cardiovascular Center at the University of Michigan for supporting this work.

\section{References}

1. Di Eusanio M, Trimarchi S, Patel HJ, Hutchison S, Suzuki T, Peterson MD, et al. Clinical presentation, management, and short-term outcome of patients with type A acute dissection complicated by mesenteric malperfusion: observations from the international registry of acute aortic dissection. J Thorac Cardiovasc Surg. 2013;145:385-90.e1.

2. Girardi LN, Krieger KH, Lee LY, Mack CA, Tortolani AJ, Isom OW. Management strategies for type A dissection complicated by peripheral vascular malperfusion. Ann Thorac Surg. 2004;77:1309-14.

3. Yagdi T, Atay Y, Engin C, Mahmudov R, Tetik O, Iyem H, et al. Impact of organ malperfusion on mortality and morbidity in acute type A aortic dissections. $J$ Card Surg. 2006;21:363-9.

4. Geirsson A, Szeto WY, Pochettino A, McGarvey ML, Keane MG, Woo YJ, et al. Significance of malperfusion syndromes prior to contemporary surgical repair for acute type A dissection: outcomes and need for additional revascularizations. Eur J Cardiothorac Surg. 2007;32:255-62.

5. Santini F, Montalbano G, Casali G, Messina A, Iafrancesco M, Luciani GB, et al. Clinical presentation is the main predictor of in-hospital death for patients with acute type A aortic dissection admitted for surgical treatment: a 25 years experience. Int J Cardiol. 2007;115:305-11.

6. Augoustides JG, Geirsson A, Szeto WY, Walsh EK, Cornelius B, Pochettino A, et al. Observational study of mortality risk stratification by ischemic presentation in patients with acute type A aortic dissection: the Penn classification. Nat Clin Pract Cardiovasc Med. 2009;6:140-6.

7. Girdauskas E, Kuntze T, Borger MA, Falk V, Mohr FW. Surgical risk of preoperative malperfusion in acute type A aortic dissection. J Thorac Cardiovasc Surg. 2009;138:1363-9.

8. Pacini D, Leone A, Belotti LM, Fortuna D, Gabbieri D, Zussa C, et al. Acute type A aortic dissection: significance of multiorgan malperfusion. Eur J Cardiothorac Surg. 2013;43:820-6.

9. Hofferberth SC, Newcomb AE, Yii MY, Yap KK, Boston RC, Nixon IK, et al. Hybrid proximal surgery plus adjunctive retrograde endovascular repair in acute
DeBakey type I dissection: superior outcomes to conventional surgical repair. $J$ Thorac Cardiovasc Surg. 2013;145:349-54.

10. Tsagakis K, Konorza T, Dohle DS, Kottenberg E, Buck T, Thielmann M, et al. Hybrid operating room concept for combined diagnostics, intervention and surgery in acute type A dissection. Eur J Cardiothorac Surg. 2013;43:397-404.

11. Leontyev S, Légaré JF, Borger MA, Buth KJ, Funkat AK, Gerhard J, et al. Creation of a scorecard to predict in-hospital death in patients undergoing operations for acute type A aortic dissection. Ann Thorac Surg. 2016;101:1700-6.

12. Grimm JC, Magruder JT, Crawford TC, Sciortino CM, Zehr KJ, Mandal K, et al. Differential outcomes of type A dissection with malperfusion according to affected organ system. Ann Cardiothorac Surg. 2016;5:202-8.

13. Deeb GM, Williams DM, Bolling SF, Quint LE, Monaghan H, Sievers J, et al. Surgical delay for acute type A dissection with malperfusion. Ann Thorac Surg. 1997;64:1669-75.

14. Williams DM, Lee DY, Hamilton BH, Marx MV, Narasimham DL, Kazanjian SN, et al. The dissected aorta: percutaneous treatment of ischemic complications_principles and results. J Vasc Interv Radiol. 1997;8:605-25.

15. Williams DM, Lee DY, Hamilton BH, Marx MV, Narasimham DL, Kazanjian SN, et al. The dissected aorta: part III. Anatomy and radiologic diagnosis of branch-vessel compromise. Radiology. 1997;203:37-44.

16. Patel HJ, Williams DM, Dasika NL, Suzuki Y, Deeb GM. Operative delay for peripheral malperfusion syndrome in acute type A aortic dissection: a long-term analysis. J Thorac Cardiovasc Surg. 2008;135:1288-95.

17. Centers for Disease Control and Prevention; National Center for Health Statistics. National Death Index. Available at: https://www.cdc.gov/nchs/ndi/index. htm. Accessed December 27, 2017.

18. Chiu P, Tsou S, Goldstone AB, Louie M, Woo YJ, Fischbein MP. Immediate operation for acute type A aortic dissection complicated by visceral or peripheral malperfusion. J Thorac Cardiovasc Surg. 2018;156:18-24.e3.

19. Midulla M, Renaud A, Martinelli T, Koussa M, Mounier-Vehier C, Prat A, et al. Endovascular fenestration in aortic dissection with acute malperfusion syndrome: immediate and late follow-up. J Thorac Cardiovasc Surg. 2011;142: 66-72.

20. Yamashiro S, Arakaki R, Kise Y, Inafuku H, Kuniyoshi Y. Management of visceral malperfusion complicated with acute type A aortic dissection. Interact Cardiovasc Thorac Surg. 2015;21:346-51.

21. Uchida K, Karube N, Yasuda S, Miyamoto T, Matsuki Y, Isoda S, et al. Pathophysiology and surgical treatment of type A acute aortic dissection. Ann Vasc Dis. 2016;9:160-7.

22. Bossone E, Corteville DC, Harris KM, Suzuki T, Fattori R, Hutchison S, et al. Stroke and outcomes in patients with acute type A aortic dissection. Circulation. 2013;128:S175-9.

23. Morimoto N, Okada K, Okita Y. Lack of neurologic improvement after aortic repair for acute type A aortic dissection complicated by cerebral malperfusion: predictors and association with survival. J Thorac Cardiovasc Surg. 2011;142: 1540-4.

24. Badreldin AM, Doerr F, Elsobky S, Brehm BR, Abul-Dahab M, Lehmann T, et al. Mortality prediction after cardiac surgery: blood lactate is indispensible. Thorac Cardiovasc Surg. 2013;61:708-17.

Key Words: acute type A aortic dissection, malperfusion syndrome, mesenteric malperfusion, endovascular fenestration/stenting, aortic surgery

\section{Discussion}

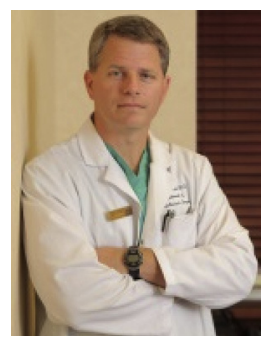

Dr Leonard N. Girardi (New York, $N Y$ ).

Thank you, Dr Yang, and congratulations to you and your colleagues at Michigan who spent really the past 2 decades trying to find a better way to treat these very-high-risk patients presenting with a type A dissection and malperfusion syndromes, and thank you for providing the manuscript in advance for my review. 
I have a number of questions. There were 82 patients who presented with mesenteric malperfusion, and those 82 underwent a total of 281 interventional procedures for an average of about 3.4 procedures per patient, and, as you saw, there were stents, fenestrations, and embolectomies.

Now, given the complexity of some of these interventional procedures, I am sure that these procedures were not completed quickly. Although you had a lot of experience, doing it over 20 years, it still has to take time to resolve some of these complex situations, and it's conceivable that perhaps some of these ultimately expiring and unresolved mesenteric ischemias could have had an opportunity to resolve their malperfusion syndrome more quickly had they gone straight to the operating room and had true lumen and end-organ flow established more expeditiously. So can you have some idea of how long it usually takes to get, from the minute they hit the door at University of Michigan, to resolution of their malperfusion?

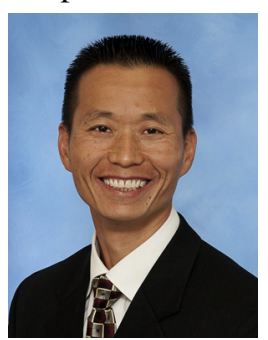

Dr Bo Yang (Ann Arbor, Mich). Dr Girardi, thank you for your question. It is a great question. We have an aortic pager at Michigan. All aortic cases come through the aortic pager; only 3 of us (aortic surgeons) are taking the call for type A dissection. When we hear that a patient has come in with abdominal pain, type A dissection, we immediately will call Dr Williams, Dr Khaja, or other interventional radiology (IR) faculty, and they mobilize the IR team.

Another advantage we have is that we can review the computed tomography scan via Life Image (Life Image Inc, Newton, Mass) before the patient was transferred and then we can see if they had a rupture or any pericardial effusion or any malperfusion of aortic branches, such as the superior mesenteric artery (SMA). So when a patient heads to the emergency department (ED), we already know the information, and after we see the patient in the ED, we move the patient from the ED to the IR suite or to the operating room (OR) pretty much in about 1 to 2 hours in recent years. This is the same in patients going to an OR for open repair or to IR for fenestration/stenting.

Actually before I came to the meeting, I asked Dr Williams, who is our interventional radiologist and performs most fenestration and stenting, about this specific question: How long does it take for you to resume the flow in the true lumen to resolve dynamic obstruction? He told me $30 \mathrm{mi}-$ nutes. He accesses the femoral artery percutaneously, puts the catheter into the true lumen, confirms the true lumen with intravascular ultrasound, pokes the dissection flap with a needle and stretches it with a balloon to create a large fenestration, and then it's done and in 30 minutes blood flow in the true lumen has resumed. That's, I think, pretty much faster than any open procedure.

And another point is, open procedure, as I mentioned, cannot resolve the static malperfusion, which is the thrombosed branch vessel, such as the SMA. We confirm the static obstruction by measuring the blood pressure at the distal SMA in the true lumen after resolving the dynamic obstruction of SMA, then we put a wire in and stent the SMA immediately. The SMA is always the first branch we treat.

Dr Girardi. Okay, thank you. At the end of the day, the goal of your strategy is to reduce the number of patients dying from mesenteric ischemia. Now, the overall mortality for the 82 patients in this series was $39 \%$, pretty sobering despite your efforts. Now, for the 47 patients who you showed had their interventional procedure and resolution of their malperfusion syndrome, those who went on to have an aortic repair did incredibly well; a mortality of $2.1 \%$ is truly impressive.

However, in the first decade of your experience, the overall mortality for those 52 patients was $40 \%-40.3 \%$ whereas in the second decade it declined to $33.3 \%$, and the majority of the reduction in that mortality was in the prevention of fatal rupture. In the first and second decades you had the same number of patients dying from mesenteric ischemia. As a matter of fact, as a percentage of the total presenting with mesenteric ischemia, a greater percentage died during the second decade.

So in addition, you had 4 patients who perhaps were unfit for open surgery and never had an operation after having their mesenteric ischemia resolved. We don't know a lot more about those patients.

So my question really is, based on your classification system for malperfusion and your imaging review, is there something that we can glean from the preoperative imaging that might tell us who would be best treated by this technique, and then, perhaps, how do we focus on those 10 in each decade who didn't survive and can we do something different? Again, is open surgery preferable for those 10 or are they too far gone?

Dr Yang. That's another great question. First, I'd like to emphasize there is a difference between mesenteric malperfusion and mesenteric malperfusion syndrome. Mesenteric malperfusion is inadequate blood flow to the intestine. The guts can be ischemic but not necrotic, especially at the early stage. Mesenteric malperfusion syndrome is the late stage of malperfusion and the intestine already has necrosis from ischemia and malfunction. During the first decade and the second decade, a total of 20 patients died from organ failure despite the resolution of SMA malperfusion. Those 20 patients all had very severe mesenteric malperfusion syndrome and malperfusion of other organs, such as kidneys, brain, and limbs, including 3 patients with necrosis of all intestines. 
Operative risk is very high in those patients. By resolving the mesenteric malperfusion and other malperfusion with endovascular fenestration and stenting, we gave those patients a chance to recover either from the resection of dead bowel, sepsis, acute respiratory distress syndrome, and other complications from mesenteric malperfusion syndrome.

We had 10 patients who died in each decade due to organ failure. The percentage of patients who died from organ failure was higher although not significant in the second decade because we have been more selective in the second decade on sending our patients for the fenestration and the stenting. We had only 30 patients who went to fenestration and stenting during the second decade and 52 patients in the first decade. The total number of patients with type A dissection were 200plus in the first decade, 300-plus in the second decade. So we have been more selective.

The preoperative computed tomography angiogram can tell us if there is malperfusion and if it is static or dynamic. However, the diagnosis of malperfusion syndrome is based on clinical presentation, such as abdominal pain, peritoneal signs, increased serum lactate, plus evidence of malperfusion on imaging. The indication of endovascular fenestration and stenting is malperfusion syndrome not malperfusion on imaging studies. Those patients who died from organ failure also had malperfusion of multiple end-organs. Any procedures, including IR or open repair, were probably futile. I think they were too far gone. Based on our studies at the angiography suite, we found out that 11 out of those 20 patients had static malperfusion that cannot be resolved by open repair. Nine patients had dynamic malperfusion because their guts were at the late stage of ischemia and necrosis. Open aortic repair could resolve the dynamic obstruction in those 9 patients but cannot reverse the necrosis and the course of their dead bowel.

Dr Girardi. My final question is the combination of stroke and mesenteric malperfusion is obviously more than most can survive and is confirmed on multivariable analysis where your odds ratio for death by organ failure was 26. Did you have an opportunity to review the computed tomography scans of those 10 patients who had the extreme bad luck of presenting with both mesenteric malperfusion and stroke to see if perhaps their neurologic impairment was secondary to cerebral malperfusion as well?

Dr Yang. Yes, they all had cerebral malperfusion at the same time.

Dr Girardi. So that would lead me to my second question then. Are there radiographic predictors that would have identified opportunities to resolve their neurologic malperfusion and their mesenteric malperfusion with an operation rather than permitting cerebral ischemia to continue while you are going to the interventional suite?

Dr Yang. That's a great question. I think you can argue to take the patient to the OR and open repair can resolve the cerebral malperfusion first, but the open aortic repair may not resolve the mesenteric malperfusion if it is static malperfusion and patients already had ongoing mesenteric ischemia and necrosis of intestine. If you have a patient with mesenteric malperfusion that had a stroke, taking those patients to the OR for open repair, I think the mortality is very, very high. I'm not sure how many patients you can save. I don't have a good answer for your question regarding which way should go first. However, In our study, those patients with cerebral malperfusion and mesenteric malperfusion syndrome did not have significant worsening of neurologic function after IR procedures. We would prefer to treat the mesenteric malperfusion first and wait for him to recover from that and see how the brain is doing, then perform central aortic repair. Another possible solution is to perform carotid artery stenting and SMA stenting for those patients during the same procedure.

Dr Girardi. Finally, whatever happened with those 4 patients?

Dr Yang. One of the patients had a stroke and was on a vent and on dialysis. He was discharged, but not too far down the road he died. Two patients survived and they are 5,6 years out and still alive without any additional operation, just medical management. There is another patient who had no stroke but had renal failure on dialysis, requiring tracheostomy and vent support and tube feeding. He didn't die from aortic rupture or dissection but eventually debilitated and died not too long after discharge.

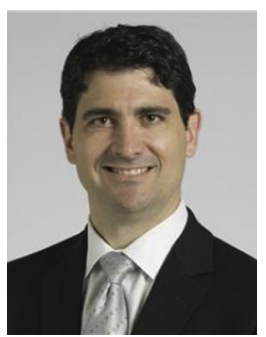
Dr Eric Roselli (Cleveland, Ohio). Bo and the team at the University of Michigan, congratulations for tackling a really difficult problem. In principle, I fully agree with the strategy that we need to fix what's going to kill the patient soonest first, and now that we have thoracic endovascular aortic repair, I think that we can address the dynamic compression although it's a little bit different than the fenestration.

But I think the area where there is a potential need for us to get better-once you have addressed the ischemia-is taking care of the rupture. Now, I've gotten burned with reperfusing a patient and high-fiving at the end of fixing an ugly ischemia problem only to have the patient rupture in that window while we are waiting.

How long do you wait once you repair the ischemia, and do you think that we should be taking all these patients to a hybrid OR, which many of us have now, if they have any signs of mesenteric ischemia so we can think about maybe getting all of those things done in 1 setting or expeditiously? 
Dr Yang. That's a great question, why wait and how long we wait. So the reasons we wait are, number 1 , we are not sure if the patient has a completely dead gut or not, and may need bowel resection. We ask general surgery to examine the patients and do a laparotomy if needed before central aortic repair. Resolving mesenteric malperfusion first helps the general surgeons better evaluate the viability of the bowel. Number 2, when the malperfusion is resolved by reperfusing the mesentery, many inflammatory cytokines, anaerobic metabolites-cellular contents from dead cells, such potassium - are released from necrotic intestine that can cause severe metabolic acidosis, acute respiratory distress syndrome, arrhythmia, and sepsis. If we operate on those patients for central aortic repair with hypothermic circulatory arrest and cardiopulmonary bypass, the mortality will be sky-high. We don't take them to the OR immediately after fenestration and stenting, nor recommend simultaneous operation at the hybrid OR. In the past 10 years, we have gained more and more experience on how to manage those patients with few patients dying from aortic rupture. We had no patients die of aortic rupture during the second decade while they were recovering from mesenteric malperfusion syndrome. We recommend waiting for patients to recover from bowel resection, metabolic acidosis, acute respiratory distress syndrome, sepsis, and septic shock and to carefully assess whether those patients they can tolerate cardiopulmonary bypass and hypothermic circulatory arrest. During the first decade, we waited much longer-weeks-for patients to fully regain bowel function and we had more patients who died from aortic rupture. During the second decade, we operated on those patients much earlier, as soon as they could tolerate cardiopulmonary bypass and hypothermic circulatory arrest. The median waiting time was about 2 days in the second decade but 4 days in the first decade for all patients with any MPS; and 4 days in the second decade but 7 days in the first decade for patients with mesenteric MPS.

So when do we operate? That's a great question. When we see the metabolic acidosis corrected, $\mathrm{pH}$ is pretty much normal, and lactate is going down, and the creatine kinase level is going down, and septic shock and acute respiratory distress syndrome are resolved. We don't wait for the kidney function to fully recover. Now, we are more actively operating on those patients within days, not waiting for 2 weeks or 3 weeks like we did during the first decade, and beyond 4 weeks we don't operate anymore.

Dr Roselli. So you wait for the lactate to clear?

Dr Yang. Not completely clear, but going down, normalizing, and we make sure there is no ongoing ischemia or necrosis.

Dr Roselli. Should we take them to the hybrid OR?

Dr Yang. Waiting gives patients an opportunity to recover from their malperfusion syndrome, and also allows surgeons to see how much damage is in the abdomen and how much bowel is dead. If surgeons do everything at the same time in a hybrid OR, including cardiopulmonary bypass, hypothermic circulatory arrest, bowel resection, that is a lot for a patient. I bet the mortality still will be very high. We prefer to wait for patients to recover from the mesenteric malperfusion syndrome, which is complications from mesenteric malperfusion, and then we do the open aortic repair as soon as patients are ready. We like our approach because it gives better chances of survival to patients who can recover from malperfusion syndrome, and also avoids futile open aortic repair for those patients who can never recover from malperfusion syndrome. 


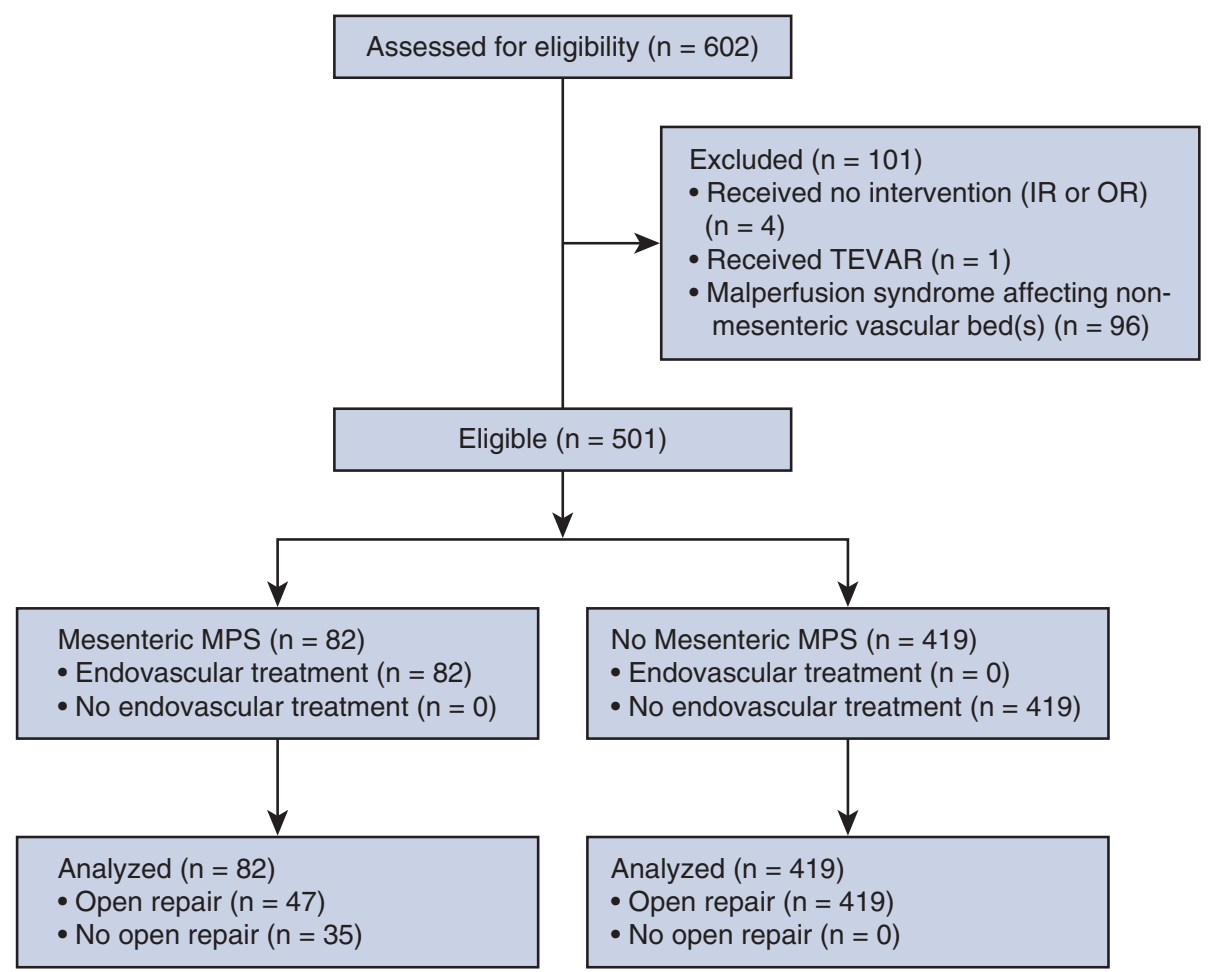

FIGURE E1. Consolidated standards of reporting trials flow diagram of patient selection. IR, Interventional radiology; OR, odds ratio; TEVAR, thoracic endovascular aortic repair; $M P S$, malperfusion syndrome.

TABLE E1. Details of endovascular interventions (82 patients treated for mesenteric malperfusion syndrome)

\begin{tabular}{lccccc}
\hline Level of aortic fenestration/stenting & $\begin{array}{c}\text { Aortic } \\
\text { fenestration }\end{array}$ & $\begin{array}{c}\text { Aortic } \\
\text { stenting }\end{array}$ & $\begin{array}{c}\text { Branch vessel } \\
\text { thrombolysis }\end{array}$ & $\begin{array}{c}\text { Branch } \\
\text { vessel suction embolectomy }\end{array}$ & $\begin{array}{c}\text { Branch vessel } \\
\text { stenting }\end{array}$ \\
\hline Descending thoracic & 0 & 1 & - & - & - \\
\hline Supraceliac & 14 & 20 & - & 0 & - \\
\hline Celiac & 16 & 0 & 0 & - & 1 \\
Supramesenteric & 30 & 44 & - & - & - \\
Mesenteric & 10 & 0 & 3 & - & - \\
Suprarenal & 3 & 3 & - & - \\
\hline Transrenal & 12 & 5 & - & - \\
Infrarenal & 28 & 46 & - & - \\
\hline
\end{tabular}


TABLE E2. Comorbidities and clinical condition on admission of patients with mesenteric malperfusion syndrome (mesMPS) or no malperfusion syndrome (non-MPS) (only patients who underwent open aortic repair)

\begin{tabular}{|c|c|c|c|c|}
\hline Variable & All patients $(N=466)$ & $\begin{array}{c}\text { MesMPS } \\
(n=47)\end{array}$ & $\begin{array}{l}\text { Non-MPS } \\
(n=419)\end{array}$ & $P$ value \\
\hline Time period & & & & $<.001$ \\
\hline First decade, 1996-2007 & $181(39)$ & $31(66)$ & $150(36)$ & \\
\hline Second decade, 2008-2017 & $285(61)$ & $16(34)$ & $269(64)$ & \\
\hline \multicolumn{5}{|c|}{ Demographic characteristics and chronic comorbidities } \\
\hline Age on admission (y) & $59(49-68)$ & $57(51-67)$ & $59(49-68)$ & .68 \\
\hline Female gender & $140(30)$ & $11(23)$ & $129(31)$ & .29 \\
\hline NYHA functional class & & & & .28 \\
\hline I or II & $344(74)$ & $40(85)$ & $304(73)$ & \\
\hline III or IV & $98(21)$ & $7(15)$ & $91(22)$ & \\
\hline CAD & $81(17)$ & $10(21)$ & $71(17)$ & .46 \\
\hline History of MI & $24(4.9)$ & $4(8.5)$ & $20(4.6)$ & .29 \\
\hline Previous cardiac surgery & $45(9.7)$ & $8(17)$ & $37(8.8)$ & .11 \\
\hline Hypertension & $326(70)$ & $40(85)$ & $289(68)$ & .017 \\
\hline COPD & $41(8.8)$ & $4(8.5)$ & $37(8.8)$ & 1.0 \\
\hline Smoking status & & & & .81 \\
\hline Never smoker & $217(47)$ & $21(45)$ & $196(47)$ & \\
\hline Former smoker & $120(26)$ & $14(30)$ & $106(25)$ & \\
\hline Current smoker & $127(27)$ & $12(26)$ & $115(27)$ & \\
\hline Diabetes & $27(5.8)$ & $2(4.3)$ & $25(6.0)$ & 1.0 \\
\hline Chronic kidney disease & $16(3.4)$ & $5(11)$ & $11(2.6)$ & .016 \\
\hline History of CVA & $14(3.0)$ & $3(6.4)$ & $11(2.6)$ & .16 \\
\hline PVOD & $55(12)$ & $7(15)$ & $48(11)$ & .49 \\
\hline Connective tissue disorder & $27(5.8)$ & $1(2.1)$ & $26(6.2)$ & .50 \\
\hline \multicolumn{5}{|l|}{ Clinical condition on admission } \\
\hline Cardiogenic shock & $35(7.5)$ & $0(0)$ & $35(8.4)$ & .038 \\
\hline Acute MI & $0(0)$ & $0(0)$ & $0(0)$ & \\
\hline Tamponade & $40(8.6)$ & $1(2.1)$ & $39(9.3)$ & .16 \\
\hline Aortic insufficiency & & & & .99 \\
\hline None & $123(26)$ & $14(30)$ & 109 (26) & \\
\hline Trace/trivial & $50(11)$ & $6(13)$ & $44(11)$ & \\
\hline Mild & $87(19)$ & $8(17)$ & $79(19)$ & \\
\hline Moderate & $77(17)$ & $8(17)$ & $69(16)$ & \\
\hline Severe & $100(21)$ & $11(23)$ & $89(21)$ & \\
\hline Acute stroke & $3(0.6)$ & $1(2.1)$ & $2(0.5)$ & .27 \\
\hline Acute paralysis & $3(0.6)$ & $3(6.4)$ & $0(0)$ & .001 \\
\hline Creatinine on admission (mg/dL) & $1.0(0.8-1.3)$ & $1.4(1.0-2.6)$ & $1.0(0.8-1.2)$ & $<.0001$ \\
\hline AKI & $53(11)$ & $35(74)$ & $18(4.3)$ & $<.0001$ \\
\hline Requiring new dialysis preoperatively & $4(0.9)$ & $4(8.5)$ & $0(0)$ & $<.0001$ \\
\hline Renal MPS & $30(6.4)$ & $30(64)$ & $0(0)$ & $<.001$ \\
\hline Lower-extremity MPS & $35(7.5)$ & $35(74)$ & $0(0)$ & $<.001$ \\
\hline \multicolumn{5}{|l|}{ Initial management } \\
\hline IR & $47(10)$ & 47 (100) & $0(0)$ & $<.001$ \\
\hline
\end{tabular}

Values are presented as median (interquartile range) for continuous variables and $\mathrm{n}(\%)$ for categorical variables. mesMPS, Malperfusion syndrome; non-MPS, no malperfusion syndrome; NYHA, New York Heart Association; $C A D$, coronary artery disease; $M I$, myocardial infarction; COPD, chronic obstructive pulmonary disease; CVA, cerebrovascular accident; $P V O D$, peripheral vascular occlusive disease; $A K I$, acute kidney injury; $I R$, endovascular procedure by interventional radiology. 
TABLE E3. Intraoperative variables of patients with mesenteric malperfusion syndrome (mesMPS) or no malperfusion syndrome (non-MPS) (only patients who underwent open aortic repair)

\begin{tabular}{|c|c|c|c|c|}
\hline Variable & $\begin{array}{l}\text { All patients } \\
(\mathrm{N}=466)\end{array}$ & $\begin{array}{c}\text { MesMPS } \\
(n=47)\end{array}$ & $\begin{array}{l}\text { Non-MPS } \\
(n=419)\end{array}$ & $P$ value \\
\hline Previous cardiac surgery* & $45(9.7)$ & $8(17)$ & $37(8.8)$ & .11 \\
\hline Aortic root procedure & & & & .60 \\
\hline None & $36(7.7)$ & $5(11)$ & $31(7.4)$ & \\
\hline Root replacement & $156(33)$ & $14(30)$ & $142(34)$ & \\
\hline Root repair & $265(57)$ & $28(60)$ & $237(57)$ & \\
\hline AVR only & $9(1.9)$ & $0(0)$ & $9(2.2)$ & \\
\hline Aortic arch procedure $\dagger$ & & & & .66 \\
\hline None & $29(6.2)$ & $1(2.1)$ & $28(6.7)$ & \\
\hline Transverse arch & $275(59)$ & $27(57)$ & $248(59)$ & \\
\hline Zone 1 arch & $36(7.7)$ & $3(6.4)$ & $33(7.9)$ & \\
\hline Zone 2 arch & $90(19)$ & $12(26)$ & $78(19)$ & \\
\hline Zone 3 arch & $36(7.7)$ & $4(8.5)$ & $32(7.6)$ & \\
\hline Frozen elephant trunk & $28(6.0)$ & $2(4.3)$ & $26(6.2)$ & 1.0 \\
\hline \multicolumn{5}{|l|}{ Concomitant procedures } \\
\hline CABG & $24(5.2)$ & $0(0)$ & $24(5.7)$ & .15 \\
\hline MV procedure & $2(0.4)$ & $0(0)$ & $2(0.5)$ & 1.0 \\
\hline $\mathrm{TV}$ procedure & $4(0.9)$ & $0(0)$ & $4(0.9)$ & 1.0 \\
\hline CPB time (min) & $219.5(179-272)$ & $222(185-261)$ & $219(177-275)$ & .77 \\
\hline Crossclamp time (min) & $154(115-199)$ & $156(127-191)$ & $154(114-201)$ & .91 \\
\hline HCA performed & $439(94)$ & $46(98)$ & $393(94)$ & .50 \\
\hline HCA time (min) & $35(28-45)$ & $38(30-46)$ & $35(28,45)$ & .2 \\
\hline Type of cerebral perfusion & & & & .65 \\
\hline ACP only & $142(30)$ & $12(26)$ & $130(31)$ & \\
\hline RCP only & $174(37)$ & $18(38)$ & $156(37)$ & \\
\hline Both ACP and RCP & $119(26)$ & $15(32)$ & $104(25)$ & \\
\hline Neither ACP nor RCP & $30(6.4)$ & $2(4.3)$ & $28(6.7)$ & \\
\hline Underwent intraoperative PRBC transfusion & $350(75)$ & $43(91)$ & $307(73)$ & .006 \\
\hline Intraoperative PRBC units & $4(1-8)$ & $7(5-10)$ & $4(1-7)$ & $<.0001$ \\
\hline 0 & $94(21)$ & $3(6.5)$ & $91(23)$ & $<.0001$ \\
\hline $1-2$ & $69(16)$ & $3(6.5)$ & $66(17)$ & \\
\hline $3-4$ & $81(18)$ & $4(8.7)$ & $77(19)$ & \\
\hline$\geq 5$ & $200(45)$ & $36(78)$ & $164(41)$ & \\
\hline
\end{tabular}

Values are presented as median (interquartile range) for continuous variables and $\mathrm{n}(\%)$ for categorical variables. Missing data points were excluded. mesMPS, Malperfusion syndrome; non-MPS, no malperfusion syndrome; $A V R$, aortic valve replacement; $C A B G$, coronary artery bypass graft; $M V$, mitral valve procedure; $T V$, tricuspid valve procedure; $C P B$, cardiopulmonary bypass; $H C A$, hypothermic circulatory arrest; $A C P$, antegrade cerebral perfusion; $R C P$, retrograde cerebral perfusion; $P R B C$, packed red blood cell. * Reoperation. $\dagger$ Transverse arch $=0$ branch vessels, zone arch $=1$ to 2 branch vessels, zone 2 arch $=2$ to 3 branch vessels, and zone 3 arch $=3$ to 4 branch vessels. 
TABLE E4. Detailed cause of death in patients with organ failure following endovascular fenestration/stenting by interventional radiology (IR), but before open repair or discharge

\begin{tabular}{|c|c|c|c|c|}
\hline Case & Age & Year of treatment & Additional sites of malperfusion & Cause of death \\
\hline \multicolumn{5}{|c|}{ First decade } \\
\hline 1 & 76 & 2000 & Renal, lower extremity & Renal failure, lower extremity necrosis \\
\hline 2 & 48 & 2002 & Renal, lower extremity & Extensive necrotic bowel \\
\hline 3 & 51 & 2003 & Cerebral, renal, lower extremity & Neurologic, renal failure, respiratory failure, shock \\
\hline 4 & 38 & 2003 & $\begin{array}{l}\text { Celiac/hepatic, renal, } \\
\text { lower extremity }\end{array}$ & $\begin{array}{l}\text { Necrotic lower extremity, shock, acidosis, renal failure, } \\
\text { respiratory failure, hepatic failure }\end{array}$ \\
\hline 5 & 56 & 2005 & Spinal, renal, lower extremity & Shock; withdrawal of care \\
\hline 6 & 74 & 2006 & Isolated mesenteric & Inoperable necrotic bowel \\
\hline 7 & 69 & 2006 & Cerebral & Stroke, arrhythmia \\
\hline 8 & 36 & 2006 & Lower extremity & $\begin{array}{l}\text { Extensive bowel necrosis, severe acidosis and hyperkalemia, not } \\
\text { compatible with life }\end{array}$ \\
\hline 9 & 80 & 2007 & Celiac/hepatic, renal & Renal failure, respiratory failure, pneumonia, withdrawal of care \\
\hline 10 & 41 & 2007 & $\begin{array}{l}\text { Cerebral, celiac/hepatic, renal, } \\
\text { lower extremity }\end{array}$ & $\begin{array}{l}\text { Large cerebral infarction, cerebral edema, brain herniation, } \\
\text { withdrawal of care }\end{array}$ \\
\hline \multicolumn{5}{|c|}{ Second decade } \\
\hline 11 & 68 & 2008 & $\begin{array}{l}\text { Cerebral, spinal, renal, } \\
\text { lower extremity }\end{array}$ & Large stroke, withdrawal of care \\
\hline 12 & 67 & 2008 & Cerebral, renal & Extensive stroke, withdrawal of care \\
\hline 13 & 35 & 2009 & Celiac/hepatic & Renal failure, liver failure, shock \\
\hline 14 & 55 & 2011 & Renal, lower extremity & Respiratory failure, renal failure, sepsis, necrotic bowel, shock \\
\hline 15 & 60 & 2011 & Cerebral, renal & $\begin{array}{l}\text { Severe large stroke, necrotic bowel, renal failure, brain } \\
\text { herniation, withdrawal of care }\end{array}$ \\
\hline 16 & 53 & 2012 & Cerebral, renal & Liver injury, sepsis, shock, diffuse brain infarction \\
\hline 17 & 90 & 2015 & Isolated mesenteric & Shock, acidosis, renal failure \\
\hline 18 & 73 & 2016 & Renal, lower extremity & Respiratory failure, renal failure, liver failure, necrotic bowel \\
\hline 19 & 43 & 2016 & Cerebral, renal & Necrotic bowel, lower extremity ischemia, arrhythmia \\
\hline 20 & 74 & 2016 & Renal, lower extremity & Bilateral lower extremity necrosis, refused amputation \\
\hline
\end{tabular}

